\title{
Percutaneous right atrial pacemaker lead repositioning using a regular deflectable ablation catheter
}

\author{
Erdem Ozel ${ }^{1}$, István Osztheimer ${ }^{2}$, Ali Ozturk ${ }^{3}$, László Gellér ${ }^{2}$, Emin Evren Ozcan³ \\ ${ }^{1}$ Cardiology Department, Tepecik Training and Research Hospital, İzmir, Turkey \\ ${ }^{2}$ Heart Center, Semmelweis University, Budapest, Hungary \\ ${ }^{3}$ Cardiology Department, Sifa University, İmir, Turkey
}

Adv Interv Cardiol 2016; 12, 2 (44): 183-184

DOI: 10.5114/aic.2016.59374

Lead dislocation is one of the most undesired complications of permanent pacemaker implantation. The rate of dislodgement of atrial pacing leads is nearly $3 \%$ [1]. Reopening the pacemaker pocket and repositioning the lead is the most commonly used technique for correcting dislocations which occur in the early period after implantation. However, this increases the risk of pocket infection and increases lead insulation problems. Snare systems and urological baskets have also been used for repositioning atrial leads [2, 3]. We report a patient who experienced dislodgement of the atrial pacemaker lead of a cardiac resynchronization therapy (CRT) device, which was corrected using a regular deflectable ablation catheter by the transfemoral route.

A 77-year-old female patient with severe ischemic left ventricular dysfunction and wide QRS had received a CRT device. At the follow-up 1 week after the procedure, atrial sensing and pacing dysfunctions were detected. Fluoroscopy revealed dislodgement of a passive fixation J-shaped atrial lead. Lead repositioning via a percutaneous transfemoral approach was planned to avoid surgical revision. A regular deflectable ablation catheter (steerable 4-mm-tip ablation catheter, Mariner, Medtronic, Minneapolis, USA) was advanced to the right atrium through a $7 \mathrm{Fr}$ introducer from the right femoral vein. We propagated the ablation catheter above the level of the atrial lead and made an anteflexion to the distal portion of the catheter. We captured the distal loop of the atrial lead, then with a slight counterclockwise rotation and pull back we oriented the atrial lead towards the right atrial appendage (Figure 1). The lead functions were normal after the intervention. The patient was discharged the next morning and had normal pacemaker functions at follow-up.

Early lead displacements after pacemaker implantations are the most frequent cause of reintervention in the majority of patients. We performed successful atrial lead repositioning with a deflectable ablation catheter by
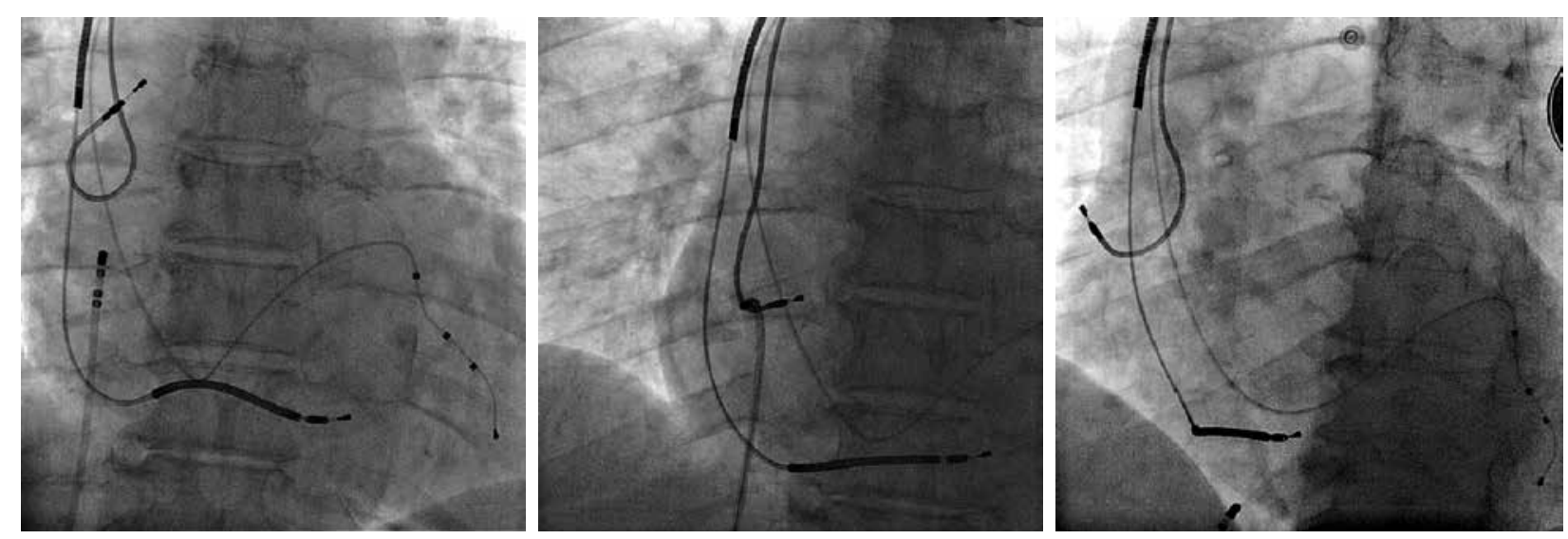

Figure 1. Successful atrial lead reposition by deflectable ablation catheter

\section{Corresponding author:}

Emin Evren Ozcan MD, Cardiology Department, Sifa University, Fevzipaşa Bulvarı No: 172/2, 35240 Basmane İzmir, Turkey, phone: +90 2324460880, e-mail: eminevrenozcan@gmail.com

Received: 11.05.2015, accepted: 30.05.2015. 
the percutaneous approach without the need for a surgical operation. However, this technique has a number of contraindications. Percutaneous lead repositioning can only be performed on patients who have passive fixation electrodes and leads with suitable loop anatomy. Conditions which necessitate the removal of the dislocated lead such as serious insulation problems are another contraindication for percutaneous lead repositioning. Repositioning of active fixation leads should be performed surgically. Since our case had a passive fixation atrial lead and a suitable loop anatomy, it was possible to perform the procedure safely. However, operators need to be cautious about dislodgement of other stable leads while performing the procedure, especially in patients with an implanted CRT. Favale et al. reported successful percutaneous atrial lead repositioning in 2 patients with permanent pacemakers and in 1 patient with an implantable cardioverter defibrillator [4]. We believe that our paper is the first one to report percutaneous atrial lead repositioning with a regular deflection ablation catheter in a patient with a CRT device.

Although it needs some experience, percutaneous transfemoral lead repositioning is a much less invasive technique and may be preferred as a first-step approach if the patient has acceptable loop anatomy of the atrial lead.

\section{Conflict of interest}

The authors declare no conflict of interest.

\section{References}

1. Kiviniemi MS, Pirnes MA, Eranen HJK, et al. Complications related to permanent pacemaker therapy. Pacing Clin Electrophysiol 1999; 22: 711-20.

2. Morris DC, Scott IR, Jamieson WR. Pacemaker electrode repositioning using the loop-snare technique. Pacing Clin Electrophysiol 1989; 12: 996-9.

3. Sharif AY, Davoodi G, Saeed AK, et al. New technique: repositioning of dislodged atrial pacing lead with a specially designed urological basket. Europace 2007; 9: 105-7.

4. Favale S, Nacci F. Percutaneous transcatheter repositioning of displaced permanent pacemaker lead. Pacing Clin Electrophysiol 1999; 22: 1817-9. 